\title{
InduCtan: a simple, robust and fast numerical tool to evalu- ate self-inductance of arbitrarily shaped coil with few wind- ings
}

\author{
Gilles MICOLAU ${ }^{1, *}$ and Elisabeth POZZO DI BORGO ${ }^{1}$ \\ ${ }^{1}$ UMR EMMAH 1114 INRA - UAPV, INRA, BP 21239 F-84916 Avignon, Cedex 9 France
}

\begin{abstract}
We built a numerical tool allowing the evaluation of self-inductance of arbitrarily shaped coils with few windings. This tool named InDUCTAN aims to be relevant, reliable and reasonably fast in order to be integrated in a more complex model. It is based on a formulation involving the vector potential and the Biot \& Savart equation. The general equation giving the self-inductance coefficient is simplified according to the hypothesis of the envisaged geometry allowing to transform a $3 \mathrm{~d}$ integral in a curvilinear integral operating on just one dimension of space. The numerical implementation is presented as exhaustively as possible, with its particular issues linked to the discrete representation of the coil. The tool is validated first on canonical geometry for which it exists an analytical formulation and second with direct experimental measurements obtained on laboratory coils with controlled and known, but not canonical, shapes.
\end{abstract}

\section{Introduction}

At the Low Noise Underground Laboratory in Rustrel [1], Luberon, France (LSBB), a conductive coil, named Vestale, lies on the top of the so-called "Grande Montagne" mountain, more than 500 metres above the tunnels of the Laboratory. It contains 12 wires with a diameter of 1.5 millimetres, forming a "screw" of few centimetre thick. Its shape is oblong, mainly determined by the topography of the site, and inscribed in a circle of a hundred metres of diameter. Vestale is currently used as an active magnetic antenna, or a source of magnetic field. It allows imaging the karstic subsoil between the top of the mountain and a superconducting magnetometer located in the underground laboratory. We project to modify Vestale to use it also as an environmental sensor based on the monitoring of its electrical characterization, possibly changing with the environment, particularly the moisture of the soil. To reach this goal, we need a numerical model for the coil self-inductance in low frequencies regime, that is to say without propagative phenomenum. It must be enough efficient to be integrated in a computation code modelling the coupling between Vestale and its environment. The evaluation of mutual or self inductance coefficients is an old and classical issue in electrodynamics [2]. The general methodology adapted to industrially produced regular shaped coils, with high density of wires, is based on the GMD [3] evaluation, and provides developped relevant analytical formulations [4] classically used, even in classroom [5]. Developments based on this methodology are still proposed [6], but at our knowledge, always for canonical shapes

\footnotetext{
*e-mail: gilles.micolau@univ-avignon.fr
} 
(the bibliography of the latest citation tends to prove that). On the other hand, heavy numerical models based on 3D finite elements method provide very reliable and relevant results for complex geometries as found in electrical engines, for instance see [7], but not relevant for our aim. Indeed, because of their complexity in term of numerical convergence and numerical cost, it is hard to integrate them in another computation code. In the more general case of antennas set near the ground interface, many theoretical and numerical developments have been done since half century, dealing with the phenomena of propagation, for instance see [8-12]. In that case, it is difficult to simply transform the model for low frequency regime without propagative terms. Our fast and cost-effective numerical tool, named InDUCTAN, is based on a semi-analytical approach allowing to extract the mathematical singularities appearing in the classical Biot \& Savart formulation. It can quickly evaluate the self-inductance of an arbitrarily shaped coil, composed of thin electrical wires, with few windings, by means of a standard desktop computer. We propose an exhaustive presentation of INDUCTAN and its validation with measurements on hand-made metric scaled coils. Then we conclude by outlining some perspectives to make INDUCTAN efficient for Vestale applications.

\section{Configuration of study}

Figure 1 shows three views of the typical coil under study. The electric device is a few

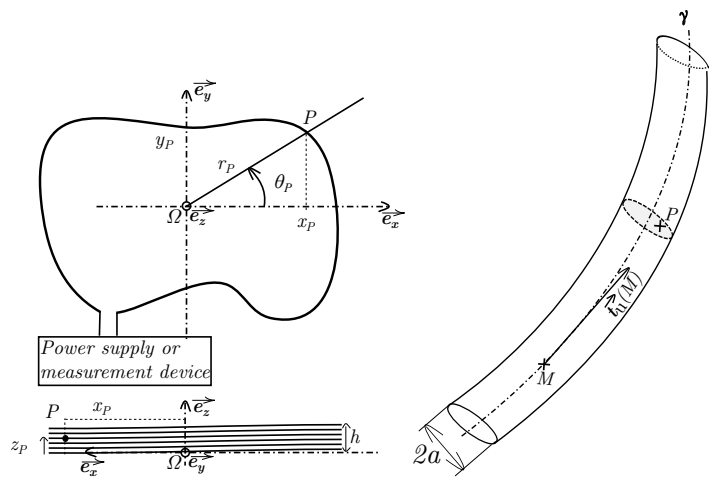

Figure 1. Top-left: top view with z axis reader oriented, bottom left: side view with y axis reader oriented, right: zoom in perspective representation of the wire. For clarity of the drawing representation, the coil is represented as an helical screw. The point $M$ is on the $\gamma$ curve, $P$ in the volume of the wire.

widings coil, obtained with a single conducting cylindrical wire, curved in space, as a cooked spaghetto. The wire is wound on itself so that the turns formed are non - contiguous. The radius of the wire is denoted $a$ supposed to be small in front of the typical size of the coil $(a<\Omega P)$, the height of the coil is denoted by $h$. The 3D curve $\gamma$ is the central axis of the wire. Each point $P$ in the volume, $\Gamma$, of the conducting wire, is spotted by cartesian coordinates $\left(x_{P}, y_{P}, z_{P}\right)$. At each point $M$ on the $\gamma$ curve, the unitary tangential vector to the curve $\gamma$ is defined and denoted by $\overrightarrow{\mathbf{t}}_{u}(M)$. In the volume of the conductor, all the points being in the same transverse section belong the same tangential vector $\overrightarrow{\mathbf{t}}_{u}$ (i.e the cross section is undeformable). The corresponding differential element of length at each point $M$ in $\gamma$ is denoted $\mathrm{d} \ell(M)=\sqrt{\mathrm{d} x_{M}^{2}+\mathrm{d} y_{M}{ }^{2}+\mathrm{d} z_{M}^{2}}$. It is associated to the elementary displacement 
vector along $\gamma, \overrightarrow{\mathrm{d} \ell}(M)$, colinear to $\overrightarrow{\mathbf{t}}_{u}(M)$. So, it comes:

$$
\overrightarrow{\mathrm{d} \ell}(M)=\mathrm{d} \ell(M) \overrightarrow{\mathbf{t}}_{u}(M)
$$

It is assumed that the coil is a perfect self inductor. Denoting by $I(t)$ the electric current flowing threw the conductor, $\dot{I}(t)$ its temporal derivative and $e_{i}(t)$ the inductive bias appearing at the terminals of the coil, the self-inductance coefficient $L$ is defined as (in electro-kinetic receiver convention)

$$
e_{i}(t)=\dot{L}(t)
$$

\section{Self-inductance formulation}

\subsection{General formulation}

We suppose the quasi-static temporal regime: the propagative terms in Maxwell's equations [13] are neglected. Moreover, taking into account the very small radius of the wire and the considered operating frequencies $(<10 \mathrm{kHz})$, it is relevant to neglect the skin effect and consider that the current is uniform in the conductor. The vector potential, $\overrightarrow{\mathbf{A}}(M, t)$ at each point $M$ in space and at every time $t$ is given by the classical relation, known as 'Biot et Savart' volumic relation [13]. Noting $S$ the cross section of the wire, (here $S=\pi a^{2}$ ) we have:

$$
\overrightarrow{\mathbf{A}}(M, t)=I(t) \frac{\mu_{0}}{4 \pi S} \iiint_{\Gamma(P)} \frac{1}{P M} \overrightarrow{\mathbf{t}_{u}}(P) \mathrm{d} \tau(P)
$$

where $\mu_{0}=4 \pi \cdot 10^{-7} \mathrm{H} \cdot \mathrm{m}^{-1}$ is the vacuum magnetic permeability

The electromotive field, $\overrightarrow{\mathbf{E}_{m}}$, is classicaly defined as the opposite of the temporal derivative of $\overrightarrow{\mathbf{A}}$ [5]. The circulation of $\overrightarrow{\mathbf{E}_{m}}$, all along $\gamma$ gives the inductive bias, $e_{i}(t)$, to which the coil is subjected.

$$
e_{i}(t)=-\int_{\gamma(M)} \overrightarrow{\mathbf{E}_{m}}(M, t) \cdot \overrightarrow{\mathrm{d} \ell}(M)=\int_{\gamma(M)} \frac{\partial \overrightarrow{\mathbf{A}}}{\partial t}(M, t) \cdot \overrightarrow{\mathrm{d} \ell}(M)
$$

Introducing then relations (2) and (3), and taking off the notations of the points of integration volume and path, we obtain the geometrical formulation for $L$.

$$
L=\frac{\mu_{0}}{4 \pi S} \int_{\gamma} \iiint_{\Gamma} \frac{1}{P M} \overrightarrow{\mathbf{t}}_{u}(P) \mathrm{d} \tau(P) \cdot \overrightarrow{\mathrm{d} \ell}(M)
$$

\subsection{D integral formulation for $L$}

The simplification of the $3 \mathrm{D}$ vectorial integration over the $\Gamma$ volume into a $1 \mathrm{D}$ integration over $\gamma$ is the theoretical core of our model. It is the key point of the numerical implementation because it contains a mathematical (and so a numerical) singularity when the points $P$ and $M$ are too close or identical. With the help of the Chasles's formula, the integration (5) can be done considering two different domains. The first one, denoted by $\Gamma \backslash M$, is constitued by the volume of the conductor from which a mesoscopic volume around the point $M$, denoted by $\delta \Gamma_{M}$, is excluded. The second one is $\delta \Gamma_{M}$. Let us denote:

$$
\overrightarrow{\mathbf{K}_{n}}(M)=\iiint_{\Gamma \backslash M} \frac{1}{P M} \overrightarrow{\mathbf{t}_{u}}(P) \mathrm{d} \tau(P) \text { and } \overrightarrow{\mathbf{K}_{s}}(M)=\iiint_{\delta \Gamma_{M}} \frac{1}{P M} \overrightarrow{\mathbf{t}_{u}}(P) \mathrm{d} \tau(P)
$$




\section{i-DUST 2018}

In the $\overrightarrow{\mathbf{K}}_{n}(M)$ integration, the distance $P M$ is a "great distance" (typically $P M>a$ ). It is supposed that, from $M$, the volume $\mathrm{d} \tau(P)$ is seen as a point, without variation of the transverse distance. So, in $\Gamma \backslash M, \mathrm{~d} \tau(P)$ is the volume of a cylinder of cross - section $S$ and length $\mathrm{d} \ell$ : $\mathrm{d} \tau(P)=S \mathrm{~d} \ell(P)$. According to relation (1), it leads to:

$$
\overrightarrow{\mathbf{K}}_{n}(M)=S \int_{\gamma \backslash M} \frac{1}{P M} \overrightarrow{\mathrm{d} \ell}(P)
$$

Concerning $\overrightarrow{\mathbf{K}}_{s}(M)$, we consider that $\delta \Gamma_{M}$ is a cylinder of length $2 \lambda$, small comparing to the total length of the wire. It is represented on figure 2 . Its central axis is supported by $\overrightarrow{\mathbf{t}_{u}}(M)$. $M$ is located at the center of the cylinder, attached at an orthonormal local coordinates system

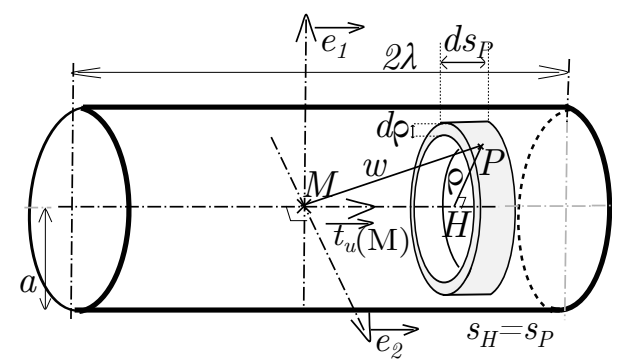

Figure 2. Scheme of the cylinder $\delta \Gamma_{M} . M$ is the center of the local system of coordinates, $s_{M}=0$. $H$ is the orthogonal projection of the point $P . w=P M, \rho=P H$. The elementary volume $d \tau(P)$ is a cylindrical tube of inner radius $\rho$, of thickness $d \rho$ and length $d s_{P}$.

$\left\{\overrightarrow{\mathbf{t}_{u}}(M), \overrightarrow{\mathbf{e}_{1}}, \overrightarrow{\mathbf{e}_{2}}\right\}$. Each point $P$ is spotted by its coordinate $s_{P}$ on $\overrightarrow{\mathbf{t}_{u}}$ axis, and its distance $\rho$ to its orthogonal projection $H$ on $\overrightarrow{\mathbf{t}}_{u}$ axis. Then $\forall P \in \delta \Gamma_{M}, P M^{2}=s_{P}^{2}+\rho^{2}$. So, $\mathrm{d} \tau(P)$ can be simplified as an elementary cylindrical tube, of inner radius $\rho$, of thickness $\mathrm{d} \rho$ and length $\mathrm{d} s_{P}$.

$$
\mathrm{d} \tau=2 \pi \rho \mathrm{d} \rho \mathrm{d} s_{P}
$$

Substituing (8) in (6), it comes:

$$
\overrightarrow{\mathbf{K}_{s}}(M)=\iint_{s_{P}} \frac{2 \pi \rho \mathrm{d} \rho}{\left(s_{P}^{2}+\rho^{2}\right)^{1 / 2}} \mathrm{~d} s_{P} \overrightarrow{\mathbf{t}}_{u}(M)
$$

Because the two variables $\rho$ et $s_{P}$ are independants, and because the primitive of the logarithm function can be defined in 0 according to the continuity definition, the integration is leaded first on $\rho \in[0, a]$, and second on $s_{P} \in[-\lambda,+\lambda]$. A very tedious but classical and straight forward calculus, using hyperbolic trigonometric variable change $\left(s_{P}=a \sinh (v)\right)$ leads to

$$
\overrightarrow{\mathbf{K}}_{s}(M)=2 \pi a^{2} F(\xi) \overrightarrow{\mathbf{t}_{u}}(M) \text { where } \xi=\frac{\lambda}{a} \text { and } F(\xi)=\ln \left(\xi+\sqrt{1+\xi^{2}}\right)+\xi\left(\sqrt{1+\xi^{2}}-\xi\right)
$$

Note that, a priori, the lambda value depends on point $M$, as the value of $\xi$. Finally, associating the relations (5), (7), (10), substituing $S=\pi a^{2}$, and noting $\gamma \backslash \lambda$ the gamma curve deprived 
of the cylinder of length $2 \lambda$, it comes an operationnal formulation for $L$.

$$
L=\frac{\mu_{0}}{4 \pi} \int_{\gamma} \int_{\gamma \backslash \lambda} \frac{1}{P M} \overrightarrow{\mathrm{d} \ell}(P) \cdot \overrightarrow{\mathrm{d} \ell}(M)+\frac{\mu_{0}}{4 \pi} 2 \int_{\gamma} F(\xi(M)) \mathrm{d} \ell(M)
$$

\subsection{Numerical formulation}

The $\gamma$ curve is parameterized uniquely by a single real variable, $v$, physically adimensionned. Each point $P(v)$ in $\gamma$ is spotted by its cartesian coordinates $\{x(v), y(v), z(v)\}$. Functions $x, y, z$ are assumed to be known, continuous and differentiable as necessary. We denote by $\dot{\mathrm{X}}$ the derivation of the quantitiy $X$ according to $v . \gamma$ curve is discretized with the help of $N_{t}$ points. We denote by an integer subscript $k$ all quantities related to the $k^{\text {th }}$ point. We denote by $\delta X$, the numerical increment of the quantity $X$ corresponding to the differential element (typically $\left.\delta X=X_{k+1}-X_{k}\right)$. Finally, denoting $F_{k}=F\left(\xi_{k}\right), L^{n}$ the numerical evaluation of the selfinductance, and for $q \neq k, D_{k q}=\left(\left(x_{k}-x_{q}\right)^{2}+\left(y_{k}-y_{q}\right)^{2}+\left(z_{k}-z_{q}\right)^{2}\right)^{-1 / 2}$, relation (11) can be numerically expressed as:

$$
L^{n}=\frac{\mu_{0}}{4 \pi} \sum_{k=1}^{N_{t}} \sum_{q \neq k} D_{k q} \delta v_{q} \delta v_{k}\left(\dot{x}_{q} \dot{x}_{k}+\dot{y}_{q} \dot{y_{k}}+\dot{z}_{q} \dot{z}_{k}\right)+\frac{\mu_{0}}{4 \pi} 2 \sum_{k=1}^{N_{t}} F_{k} \delta \ell_{k}
$$

With this numerical formulation, the value of $\lambda_{k}$ is $\lambda_{k}=\delta \ell_{k}$. That is to say the number of points $N_{t}$ is the single numerical parameter conditionning the computation. Roughly, if $\ell$ is the total length of the coil, $\lambda_{k}=\ell / N_{t}$. If $N_{t}$ is too small, the geometry of the coil is numerically ill-represented. On the other hand, if it's too high, the hypothesis allowing to calculate $\overrightarrow{\mathbf{K}}_{n}$ as an integral just over the $\gamma$ curve could becomes invalid. $N_{t}$ has to be adjusted for correct evaluation of $L^{n}$. As it is often the case in statics domain, there is not a reference length (as the wavelength could be in radio-frequency regime). That is to say, it is difficult to have an a priori value of $\lambda_{k}$ (i.e $N_{t}$ ) ensuring a good convergence of the computation. Practically, a convergence benchmark versus $N_{t}$ has to be executed for each evaluation of $L^{n}$.

\section{Validations}

We detail here two instances of validation, the first one comes from the bibliography [4] and allows to present the issues concerning the domain of validity of our numerical formulation. The second instance is an experimental validation.

\subsection{Circular ring with circular section}

In the case studied in [4], $\gamma$ curve is a circle of radius $R_{0}=0.69 \mathrm{~m}$, the radius of the wire being $a=1.5 \mathrm{~mm}$. The figure 3 shows the $L^{n}$ values generated by our model versus $N_{t}$ (left) and versus $\xi=2 \pi R_{0} /(a(N t-1))$ (right) wich is constant along the circle, in this particular case. $L^{n}$ reaches a minimum value of $L^{n}=5,719 \mu \mathrm{H}$ for $40 \geq N_{t} \leq 80$. This is a stability zone where $L^{n}$ is no longer sensitive to $N_{t}$ variations. Outside this zone, the computation is not stable, decreasing before, increasing after. The interpretation of this behavior is the following. When $N_{t}$ small $(\leq 40)$, there are not enough points $M_{i}$ to correctly discretize the geometry of the ring, the magnetic influence of each part of the coil to the others is not correctly taken into account. On the other hand for $N_{t}$ high $(\geq 80)$, there are too many points to ensure the approximation allowing the truncation of the integral leading to equation (11): 

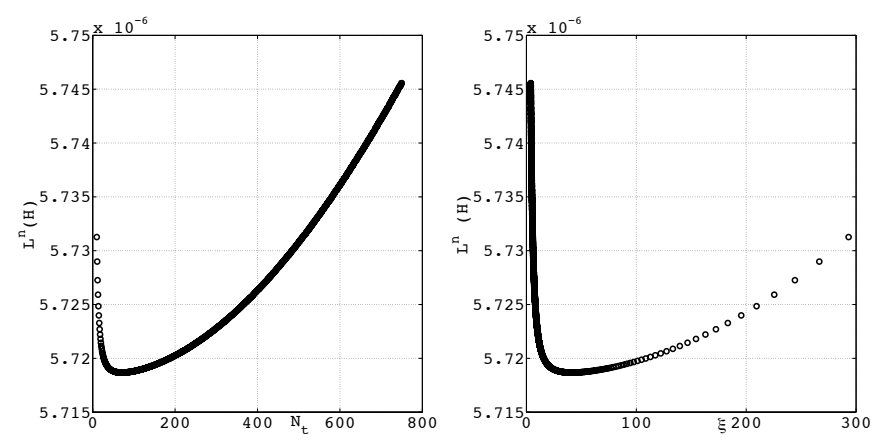

Figure 3. Estimated value $L^{n}$ for circular ring of radius $R_{0}=0,69 \mathrm{~m}$ and cross section radius a $=1.5$ mm versus $N_{t}$ (left) or $\xi$ (right).

the $M_{i}$ points are too close to each other. The self-inductance, $L_{a}$, of such a ring is given in [4], page 143, relation (119b), or [5], page 173, beside figure 203. It is reported in relation (13), with the numerical apply with our value.

$$
L_{a}=4.10^{-3} \pi R_{0}\left[\ln \left(\frac{8 R_{0}}{a}\right)-1,75\right]=>L_{a}=5,60 \mu \mathrm{H}
$$

where $L_{a}$ is expressed in $\mu H$, a and $R_{0}$ are in centimeters.

We denote a relative difference around $2 \%$ between the admitted formula and the result given by INDUCTAN in the stability zone. This value has been remarked for several benchmarks on INDUCTAN, including the evaluation of the self-inductance for a straight wire.

\subsection{Experimental validation}

We built a metre-scale mock-up, in the laboratory to explorate the possibilities of different configuration settings of interconnexions of the wire in Vestale and to study the sensitivity of direct measurements with different materials around the coil. The mok-up is built in view to be filled with water, or sand or both. There is absolutely no metal part in it. It is totally made with wood and glue. Wood was chosen as buiding material because of its almost absence of magnetic properties and its solidity. Another materials as mineral or organic glass would have been too expansive and too fragile. It is difficult to precisely determine the influence of the wood because the coil must be fixed on a rigid support to have a reproductible geometric shape. Then it is quite impossible to have some reference measurement in vacuum for a given shape.

Nevertheless, according that wood is completely non magnetic $\left(\mu_{r}=1\right)$, we used it to directly measure the self-inductance of a coil weirdly shaped. Mockup photographies are presented in figure 4. The first step of the process consists in describing mathematically the shape of the coil. It is described by the help of a Fourier serie in polar coordinates, in the plane of the view of the figure 4. The coefficients are extracted by an image treatment of the photography of the coil. Because we do not know exactly how the wire is arranged in the plastic sheath, we assume that the coil follows an helical screw of 12 loops on the $z$ axis (coming to the reader), of heigth $h$, with constant vertical step $(h / 12)$ and a radius $a=0.69 \mathrm{~mm}$ (manufacturer's 


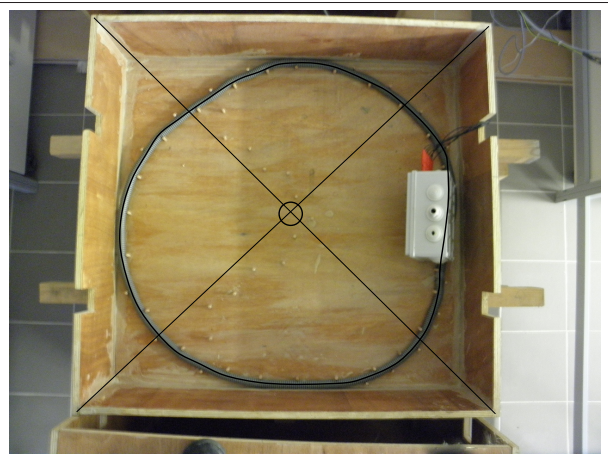

Figure 4. Laboratory mockup of Vestale. The coil is composed of 12 wires lying in the plastic grey sheath, interconnected by a set of terminals in the white plastic box right in the wood box. The box is a square of 1 metre side. We can configure the interconnexions by a set of swithing devices for having all loops (12), just 6, or just 1. The wire is of radius $a=0.69 \mathrm{~mm}$. The two terminals of the coil are related to a RLC metre Keysight E4980, working in harmonic regime from $20 \mathrm{~Hz}$ to $300 \mathrm{kHz}$. The coil has two possible reproductible shapes, just the one ("potatoe shape") used for the validation is shown here.

data). In the figure (5), left, are presented histograms of the direct measurements of the potatoe shape for four operating frequencies. It appears that the mean value for all frequencies
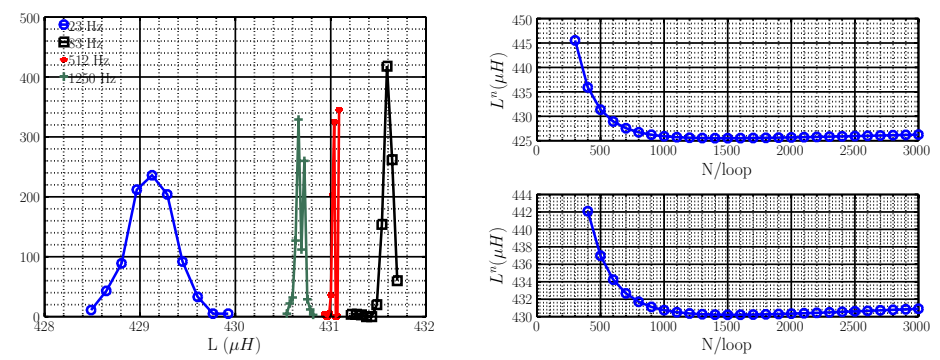

Figure 5. Left:Histograms of the measured self-inductance of the potatoe shape for four different frequencies $(23,83,512$ and $1250 \mathrm{~Hz})$. Right top: $L^{n}$ versus the number of points of discretization $N /$ loop for $h=20 \mathrm{~mm}$ and $a=0.69 \mathrm{~mm}$, right bottom: $L^{n}$ versus the number of points of discretization $N /$ loop for $h=18.9 \mathrm{~mm}$ and $a=0.69 \mathrm{~mm}$.

is $430 \mu \mathrm{H}$, with variability around $0.5 \%$ ([428 - 432] $\mu \mathrm{H})$. It is reasonable to aim this target value with InDuctan. In the right of the figure (5) are presented the results provided by INDUCTAN versus the number of points by loop, with two set of numerical entries for $h: h=$ $20 \mathrm{~mm}$ (measured diameter of the plastic sheath) on top, an $h=18.9 \mathrm{~mm}$ bottom. We remark that InDUCTAN gives stable results in a zone of $N / m$ value going from 1000 points/loop up to 2000 points/loop. The minimum value provided by INDUCTAN is $L^{n}=425 \mu H$ (top) with numerical entries corresponding to the measured height $h$. It is around $1 \%$ different from the direct measured data. This difference can be explained by two complementaries ways. The first one lies in the difference between the real geometry of the coil in the plastic sheath: the wire does not fill all the volume of the sheath and moreover, we do not know exactly the actual 
geometry of the wire inside it. To explore this interpretation, we modified the numerical entry for $h$ at $18.9 \mathrm{~mm}$. This is the bottom curve in the right of the figure 5. We notice that, with this value, the target of $430 \mu \mathrm{H}$ is reached. The second interpretation of the difference could be that we did not take into account the self-inductance of the connecting wires between the RCLmeter and the coil. The classical formula of self-inductance of a straight wire, [4], page 35 , Eq. (7), provides a value around $\sim 2.3 \mu H$ for a length $\ell=1.5 \mathrm{~m}$ and radius $a=0.69 \mathrm{~mm}$. Because we have two connecting wires measuring $\sim 1.5 \mathrm{~m}$, reasonnably separed from each other, we can evaluate roughly the connecting inductance around $2 \times 2.3=4.6 \mu H$. That is compatible with the difference between measured and numerically evaluated value of $L$ for the potatoe shape.

To finish this paragraph, let us notice that for the last evaluation on the right curves of figure 5, corresponding to 3000 points/loop (i.e $N_{t}=36000$ ), the computation time is around 40" on a standard CPU desktop computer and the memory need is around 14Go of RAM, while the point corresponding to the stability zone (1500 points/loop i.e $\left.N_{t}=18000\right)$, computation time is around 20" with 4Go of RAM. The code has been developed on the free open source platform OcTAVE GNU.

\section{Vestale application issues}

Vestale shape has been numerically modelled with the same methodology as the mockup (see section 4.2). Several hundred of points have been landmarked, with the help of the GPS, and their coordinates have been reported in an arbitrarily cartesian system. The Fourier serie modelling the shape is computed as explained in section 4.2. The coil is then modelled as an helicoidal coil of height $h \sim 20 \mathrm{~mm}$, with 12 widings. In that case of study, the current version of INDUCTAN fails to compute, in the way we want, the self-inductance. The stability zone of $L^{n}$ versus $N_{t}$ cannot be reached before overflowing the RAM of our machine (64 Go RAM). This is due to the fact that for a given point $M_{k}$, in the numerical representation of Vestale, the nearest point is not $M_{k \pm 1}$ in the same loop but a point $M_{k \pm 1 L}$ ( $1 L$ denotes symbolically 1 loop), located in the loop just above or just bottom. To emphasize this point, we give a rough numerical evaluation of the needed RAM to ensure convergence.

The order of magnitude of the length of 1 loop of Vestale is $300 \mathrm{~m}$. The order of magnitude of $M_{k} M_{k \pm 1 L}$ is around $h / 12$, ie: $M_{k} M_{k \pm 1 L} \sim 1.8 \mathrm{~mm}$. To reach the stability zone, our numerical experience on mockups leads to consider that the order of magnitude of $M_{k} M_{k \pm 1 L}$ has to be the same as $M_{k} M_{k \pm 1}$. With 12 loops, the total needed number of points $N_{t}$ is also around

$$
N_{t} \sim 12 \times \frac{300}{1.8 \times 10^{3}} \sim 2 \times 10^{6} .
$$

The number of elements, $N_{D}$, of the matrix containing $D_{k q}$, defined in 12 , is around $N_{t}^{2} \sim$ $4 \times 10^{12}$. Encoding the floating number in a low 8-bit format, $N_{D}$ is the order of magnitude of the RAM needed to ensure the computation. It is greater than 1 To of RAM. It is not yet a standard device on desktop computer.

Our interpretation of the failure of the current version in computing self-inductance of Vestale is confirmed by the fact that INDUCTAN provides converging results for the evaluation of the self inductance for just 1 loop of Vestale, in the same numerical conditions as the mockups validation ones.

Because the main idea of INDUCTAN is to provide, robust, fast and light numerical formulation, we don't aim to buy RAM and super-computers to overpass this difficulty. Two ways are under study for optimizing the numerical formulation. The first one consists in trying to decrease the needed number of points integration method by weighting points as Gauss one 
for instance [14]. The other one, more promising in term of extension of number of loops is two compute the self-inductance of 1 loop and the mutual cross inductance between all the loops.

\section{Conclusion}

We have presented the theoretical and the numerical implementation of a numerical tool, named INDUCTAN, allowing evaluation of arbitrarily coil shaped self-inductance. Some issues of the numerical process have been discussed with the help of validating tests using direct measurements. INDUCTAN is well adapted for aspect ratios coils corresponding with meter scaled coils, with radius wire and interspace typically around the millimeter size. In the very next future, it will be usefull to find empiric criteria for avoiding convergences tests a posteriori of the calculation versus $N_{t}$, using parametric studies, and involving the other possible shape of the mock-ip coil. The challenge will be then to adapt INDUCTAN for larger coil, with typical size of hundred meters as Vestale is. In its current formulation, it needs too many points of discretization of the coil to provide reliable results for such a coil. To reach this goal, some theoretical reformulations have to be done, particularly approximations about consecutive loops interactions. Then in the far future, it will be great to integrate InDUCTAN in a complete model taking into account the ground interface.

\section{References}

[1] http://www.lsbb.eu

[2] J. C. Maxwell, A treatise on electricity and Magnetism, Vol II, (Oxford Clarendon Press, 1873), 516 pages.

[3] R. Weaver. Geometric Mean Distance - An Evaluation of its Accuracy for Inductance Calculations.

(2017) 10.13140/RG.2.2.24414.23367, http://electronbunker.ca/DLpublic/GMD.pdf

[4] F. W. Grover, Inductance Calculation, (Dover, New York, 2009 rep 2004, first Ed 1946) 286 pages.

[5] Y. Rocard, Electricité,(Masson, Paris, 1951), 538 pages, pp 173.

[6] S. Babic, F. Sirois, C. Akyel, C. Girardi, IEEE Trans. Magnetics, Vol 460, No 9, pp 35913599, (Sept. 2010)

[7] A. Foggia, techniques de l’Ingénieur, D3440-V1, pp D3440 1- 20, (Fev. 1999)

[8] T. J. Cui, W. C. Chew, IEEE Trans. Antennas and Propagation, Vol 50, No 3, pp 301-307, (March 2002)

[9] T. J. Cui, W. C. Chew, IEEE Trans. Antennas and Propagation, Vol 48, No 4, pp 482-493, (April 2000)

[10] G. J. Burke, E. K. Miller, IEEE Trans. Antennas and Propagation, Vol AP-32, No 10, pp 1040-1049, (Oct.1984)

[11] C. M. Butler, D. R. Wilton, IEEE Trans. Antennas and Propagation, succint papers, pp 534-540, (July 1975)

[12] R. King, C. W. Harrison, Proceedings of the I.R.E, pp 548-567, (Oct. 1943)

[13] J. D. Jackson, Classical Electrodynamics,(Wiley Sons New York, 1975, 2nd Edition), 848 pages, Chapters 5, 6 .

[14] M. J. Yielding, Disk ray theory in transversaly isotropic media, PHD Thesis, University of British Columbia, Vancouver (Canada), Department of Geophysics and Astronomy, April 1978 and private discussions 